\title{
Otimização das cirurgias em um hospital universitário: avaliação do tempo en tre o período pré-anestésico e o momento da incisão cirúrgica
}

\author{
Solange M. Laurencel,${ }^{1{ }^{*}}$ Bruno B. P. Madruga, ${ }^{1}$ Fabiana Lucia L. Carvalho, ${ }^{1}$ Marcelo S. Duran ${ }^{1}$
}

\begin{abstract}
Resumo
O objetivo primordial do trabalho foi verificar se os médicos em especialização pertinentes às clínicas cirúrgicas do Hospital Universitário Pedro Ernesto (HUPE) têm noção do tempo decorrido entre o preparo anestésico até o período de início da cirurgia, assim como as consequências que poderão advir para o paciente. Entre as mesmas, é possível citar: hipotermia, infecção, distúrbios da coagulação e aumento na permanência hospitalar. No decorrer do estudo, constatou-se que vários fatores influenciavam o tempo entre preparo e a execução: material para realização da cirurgia, espera pelo preceptor, liberação dos hemoderivados, posicionamento do paciente na mesa de cirurgia, limpeza da sala de cirurgia e procedimentos anestésicos de difícil execução. A taxionomia obtida permitiu verificar a validade do objetivo proposto. Tem-se como conclusão final que a otimização das cirurgias no HUPE implica no aumento da interação dos diferentes atores presentes no processo anestésico cirúrgico. Esta sinergia aprimora a qualidade dos serviços cirúrgicos, especialmente os de alta complexidade, garantindo, de forma ampla, a segurança dos pacientes do HUPE quando da execução do ato cirúrgico. Para alcançar a plenitude da otimização, faz-se necessário o monitoramento constante por meio da construção de indicadores, check-liste dispositivos de vigilância com vistas à obtenção de ótima performance.
\end{abstract}

Descritores: Otimização; Tempo cirúrgico; Monitoramento; Complicações.

\section{Abstract \\ Optimization of surgery in a university hospital: evaluation of time between the pre-anesthetic pe- riod and the timing of the surgical incision}

The main objective of the research was to verify if residents specialist in in clinical surgery from Pedro Ernesto University Hospital (HUPE) have acquaintance of the time elapsed between anesthetic preparations and beginning of surgery and also of its consequence. We can cite: hypothermia, infection, coagulation disorders and increase in hospital stay. During the study, it was found that several factors influenced time between preparation and implementation of surgery: material for surgery execution, waiting time for preceptor, release of blood products, positioning the patient on surgery table, cleanliness of the place for surgery and surgery procedures for difficult anesthetic execution. Taxonomy obtained allowed to check the validity of proposed objective. As final conclusion optimization of surgery in HUPE imply upgrade
1. Serviço de Anestesiologia. Departamento de Cirurgia Geral. Hospital Universitário Pedro Ernesto. Universidade do Estado do Rio de Janeiro. Rio de Janeiro, RJ, Brasil.

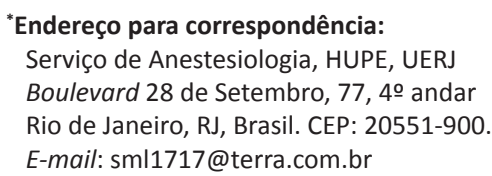

Revista HUPE, Rio de Janeiro, 2015;14(2):11-16

doi:10.12957/rhupe.2015.18319

Recebido em 25/11/2014. Aprovado em 09/01/2015.

of different actors' interaction on surgical anesthetic process. This synergy improves surgical services quality, especially on high complexity surgeries, ensuring, broad form, and security of HUPE patients when surgeries are done. Aiming to obtain full optimization, it is necessary constant monitoring, by construction of indicators checklist and vigilance devices, in order to obtain optimal performance.

Keywords: Optimization; Surgical time monitoring; Complication.

\section{Resumen}

Optimización de la cirugía en un hospital universitario: evaluación de tiempo entre el período pre-anestésica y el momento de la incisión quirúrgica

El objetivo principal de este estudio fue determinar si los médicos que asisten a la especialización en clínicas quirúrgicas del Hospital Universitario Pedro Ernesto (HUPE) tienen pista de tiempo transcurrido entre la preparación anestésica para el período inicial de la cirugía, y las consecuencias que puedan derivarse para el paciente. Entre ellos, se puede mencionar: la hipotermia, infecciones, trastornos de la coagulación y el aumento de la estancia hospitalaria. Durante el estudio, se encontró que varios factores influyeron en el tiempo entre la preparación y la ejecución es el material para realizar la cirugía, a la espera para el profesor, la liberación de los productos sanguíneos, la posición del paciente en la mesa de operaciones, limpieza de la sala de operaciones y procedimientos anestésicos difícil de implementar. La taxonomía obtenidos permitieron verificar la validez del objetivo propuesto. Ha sido la 


\section{Artigo original}

conclusión final de que la optimización de la cirugía en HUPE implica el aumento de la interacción de los diferentes actores presentes en el proceso anestésico quirúrgico. Esta sinergia mejora la calidad de los servicios quirúrgicos, especialmente los de alta complejidad, lo que garantiza en términos generales, la seguridad de los pacientes Hupe cuando se realiza la cirugía.

\section{Introdução}

É sabido que a permanência do paciente no centro cirúrgico tem algumas consequências, como a exposição a baixas temperaturas. Esta exposição pode levar à hipotermia ${ }^{1,2} \mathrm{e}$, consequentemente, atrasar o despertar ${ }^{3}$ e aumentar a possibilidade de distúrbios de coagulação, ${ }^{4}$ reduzindo a resposta imunológica e aumentando a infecção. ${ }^{5}$ Assim, em hospitais de grande porte, como o Hospital Universitário Pedro Ernesto (HUPE), é importante que se identifiquem fatores que levam ao aumento do tempo de permanência no centro cirúrgico para que se encontre meios para otimizar esse tempo frente à instituição.

O objetivo do trabalho foi verificar se os novos médicos em especialização das clínicas cirúrgicas do HUPE têm noção sobre o tempo decorrido entre o início do preparo anestésico até o momento da incisão cirúrgica e, também, da importância deste tempo na recuperação dos pacientes.

\section{Desenvolvimento}

Um questionário foi elaborado para ser respondido pelos médicos em especialização e seus respectivos preceptores, que assinaram também um termo de con-
Para alcanzar la plenitud de la optimización, es necesario un seguimiento constante mediante la construcción de indicadores, lista de control y dispositivos de vigilancia con el fin de obtener un rendimiento óptimo.

Palabras clave: Optimización; El tiempo quirúrgico; Monitoreo; Complicaciones.

sentimento livre e esclarecido (TCLE). De uma forma geral, as perguntas feitas foram sobre: a preocupação da equipe quanto à demora do início da cirurgia, a estimativa de tempo para o início da cirurgia (incisão cirúrgica após a liberação pela equipe anestesiológica), o conhecimento das complicações e suas consequências para o paciente cirúrgico. Além disso, foi solicitado que fornecessem sugestões para otimizar o tempo e, consequentemente, reduzir o período de permanência do paciente no centro cirúrgico.

Foram analisadas 48 cirurgias eletivas e configuradas no mapa normal do hospital, distribuídas pelas seguintes especialidades: cirurgia geral, urologia, cirurgia pediátrica (CIPE), cirurgia vascular, ginecologia, otorrinolaringologia, neurocirurgia, ortopedia e proctologia (Figura 1).

Em cada uma das cirurgias, registrou-se o horário desde a entrada no centro cirúrgico para o preparo anestésico até o momento da incisão cirúrgica. Na tabela 1, temos a distribuição do tempo total das 48 cirurgias nas quatro etapas principais do procedimento.

A análise dos dados identificou os seguintes motivos da demora referente ao procedimento anestésicocirúrgico (Figura 2): problemas com materiais, espera do preceptor de cirurgia, liberação de hemoderivados,

Figura 1. Distribuição, por especialidade, das 48 cirurgias analisadas.
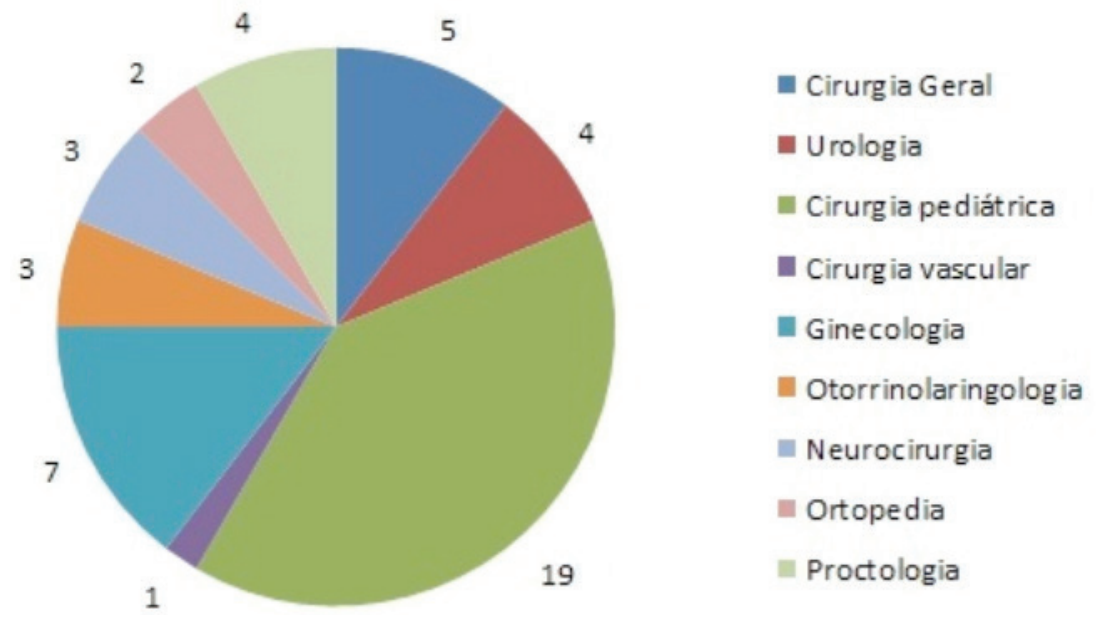
posicionamento do paciente, limpeza da sala, procedimentos difíceis e problemas burocráticos do hospital (greve). Cabe salientar, entretanto, que a maioria dos médicos participantes do nosso trabalho está no início de sua formação cirúrgica (especializando-se).

Para otimizar o tempo entre a chegada do paciente ao ambiente cirúrgico e o real início da cirurgia, os dados sugeriram as seguintes ações:

- melhor organização entre as equipes de cirurgia e anestesiologia;

- agilização do encaminhamento do paciente ao centro cirúrgico;

- entrada na sala de cirurgia o mais cedo possível, com limite de horário às 07h30 (normalmente as atividades no centro cirúrgico no HUPE se iniciam às 8 h);

- verificação pelo plantão noturno da viabilidade de sangue, material cirúrgico e vagas em unidades fechadas, evitando a solicitação de um paciente desnecessariamente;

- agilização da limpeza das salas entre as cirurgias. Quanto à preocupação com o início de uma cirurgia, houve uma divergência entre as respostas dos preceptores e dos médicos em especialização. Estes últimos preocupam-se em não atrasar a cirurgia para que não haja um retardamento na conclusão do mapa cirúrgico da referida especialidade, ou seja, querem "operar mais". Em outras palavras, esses médicos não têm uma preo-

Figura 2. Motivos da demora no procedimento anestésico-cirúrgico.
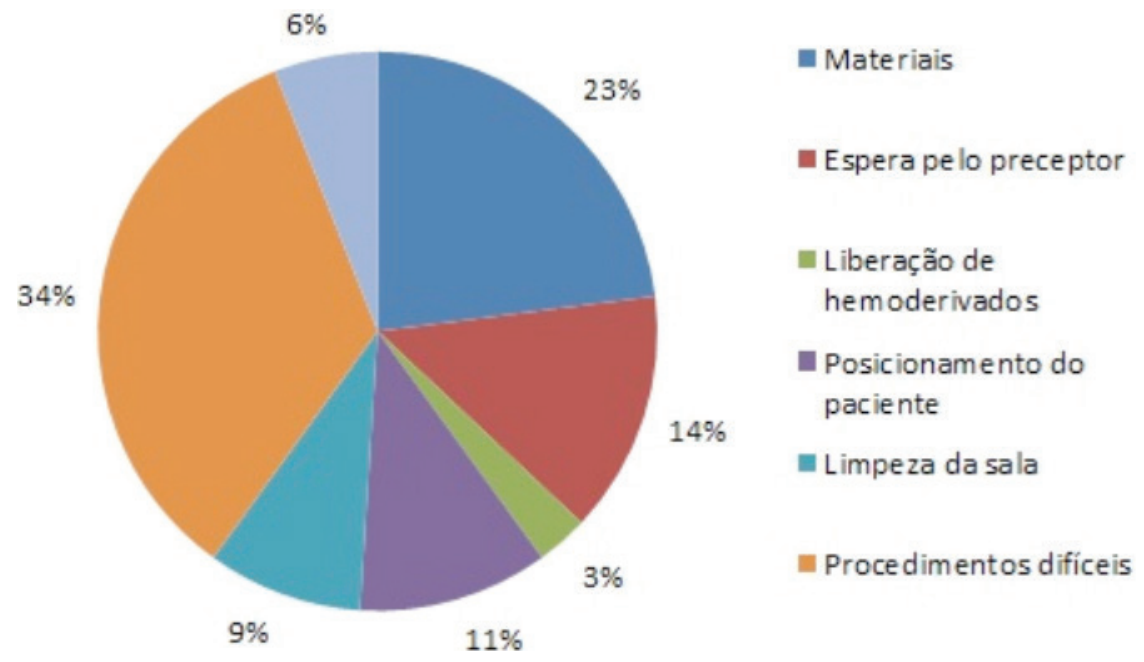

Figura 3. Compração das distribuições dos tempos real e estimado.

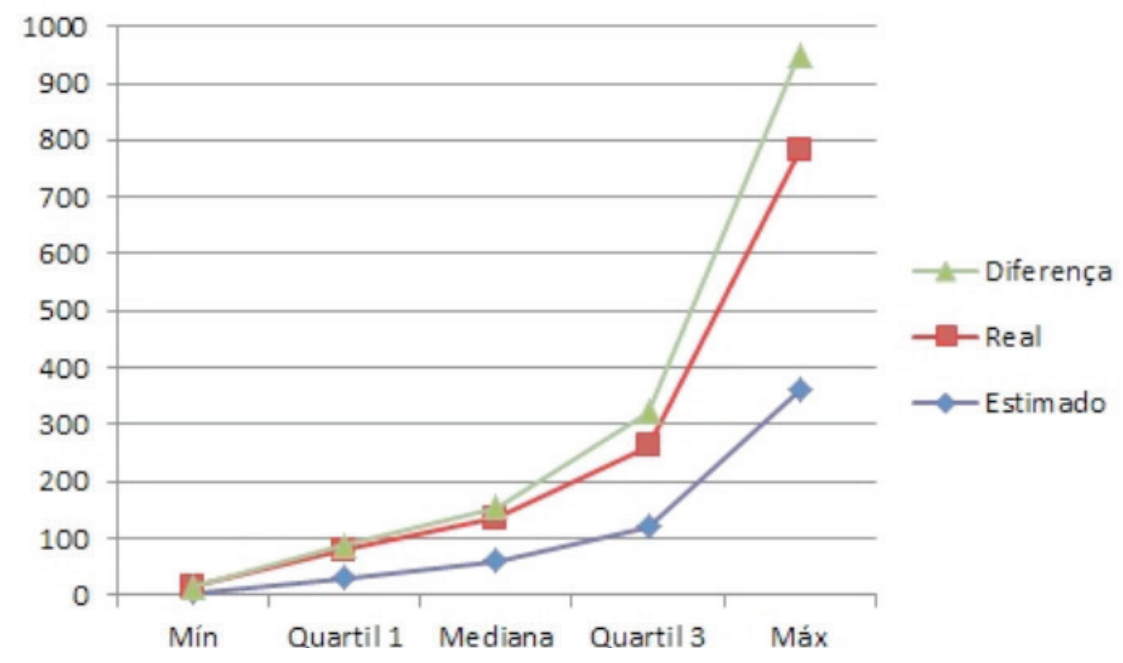




\section{Artigo original}

cupação direta com o aumento do tempo do paciente no centro cirúrgico. No entanto, essa é uma questão importante entre os preceptores, que demonstram suas preocupações quanto ao aumento do risco de infecções, desenvolvimento de distúrbios eletrolíticos, trauma dos pacientes e familiares e o próprio desgaste físico e mental da equipe. Essa divergência nas respostas mostra claramente a maior maturidade do médico preceptor com mais de cinco anos de experiência em relação ao médico em especialização.

Aproveitando o questionário que gerou nossa pesquisa, podemos avaliar outros parâmetros importantes na otimização do atendimento aos pacientes cirúrgicos. O tempo entre a solicitação do paciente e a sua chegada no centro cirúrgico levou em média 20 minutos. Os pacientes normalmente são encaminhados à sala de recuperação pós-anestésica (RPA), para reavaliação do estado físico e laboratorial do paciente, como também é realizada a canulização de acesso venoso periférico. A higienização da sala cirúrgica é outro fator importante que gera demora no começo da cirurgia, principalmente nas cirurgias subsequentes, o que aumenta a chance de suspensões de cirurgias programadas para o final da tarde. Esse fato gera um prejuízo no aprendizado dos médicos em especialização. Outro motivo observado foi a demora na liberação de material cirúrgico pela central de material do hospital, que causou uma espera de aproximadamente 60 minutos, sendo em média 40 minutos para o início da cirurgia.

Com relação à duração do ato cirúrgico em si, para apenas cinco das 48 cirurgias levantadas, houve concordância entre o tempo cirúrgico estimado pelo cirurgião e o tempo real gasto no procedimento. A

Tabela 1. Distribuição do tempo total das 48 cirurgias nas quatro etapas principais.

\begin{tabular}{lcc}
\hline \multicolumn{1}{c}{ Etapa } & \multicolumn{2}{c}{ Tempo decorrido } \\
& Minutos & $\%$ \\
\hline $\begin{array}{l}\text { Da solicitação à chegada do } \\
\text { paciente no centro cirúrgico }\end{array}$ & 788 & 15,0 \\
\hline $\begin{array}{l}\text { Da chegada ao centro } \\
\text { cirúrgico à entrada em sala } \\
\text { operatória }\end{array}$ & 1.261 & 24,0 \\
$\begin{array}{l}\text { Do início da anestesia à } \\
\text { liberação do paciente para o } \\
\text { cirurgião }\end{array}$ & 1.102 & 21,0 \\
\hline $\begin{array}{l}\text { Cirurgia } \\
\text { Total }\end{array}$ & 2.101 & 40,0 \\
\hline
\end{tabular}

tabela 2 mostra que, em média, o tempo estimado é maior, apresentando uma menor variabilidade quando comparado com o tempo real. Na figura 3, podemos observar que existe um aumento na diferença à medida que aumento o tempo real da cirurgia.

Mesmo quando não ocorreram complicações cirúrgicas, este tempo por vezes ultrapassou em duas horas do estimado. Não encontramos na literatura trabalhos específicos que estimassem o tempo cirúrgico gasto para compararmos com os nossos resultados. Torna-se sensato que, por estarmos em uma instituição de ensino, o tempo cirúrgico devesse ser mais prolongado.

\section{Discussão}

Podemos observar que nos dias atuais é exigida uma qualificação cada vez maior de todos profissionais ativos no mercado de trabalho. Esse fato é mais intenso quando tratamos de profissionais da área de saúde, principalmente médica. Essa exigência em aperfeiçoamento é materializada na classe médica com o curso de especialização na clínica escolhida. Assim, há exigência de grandes centros de residência médica com hospitais de múltiplas especialidades que se tornam referência regional em educação e saúde.

O HUPE não foge à esta exigência da atualidade. Inaugurado em 1950, tornou-se hospital-escola da Faculdade de Ciências Médicas da Universidade do Estado da Guanabara, atual Universidade do Estado do Rio de Janeiro. Em 1975, torna-se um hospital de atendimento geral em acordo com o Ministério da Educação. Devido à qualidade de seus profissionais e aos meios sofisticados de diagnóstico e tratamento que são oferecidos, o HUPE transformou-se em um dos maiores complexos docente-assistenciais na área de saúde, além de ser considerado referência em uma série de especialidades e um importante núcleo nacional de formação

Tabela 2. Comparação do tempo real e o tempo estimado (em minutos) das cirurgias.

\begin{tabular}{lcc|c}
\hline & $\begin{array}{c}\text { Tempo } \\
\text { estimado (E) }\end{array}$ & Tempo real (R) & $\begin{array}{c}\text { Diferença } \\
|\mathbf{R}-\mathbf{E}|\end{array}$ \\
\hline Média & 85,1 & 113,9 & 38,7 \\
\hline Desvio-padrão & 71,2 & 101,9 & 42,9 \\
\hline Moda & 60,0 & 30,0 & 0 \\
\hline Mediana & 60,0 & 75,0 & 20 \\
\hline
\end{tabular}


de profissionais de saúde. Atualmente, funciona com 525 leitos e mais de 60 especialidades e subespecialidades, acolhendo internos, especializandos e residentes. As especialidades cirúrgicas que compõem o HUPE correspondem a aproximadamente $55 \%$ dos leitos oferecidos, destacando-se, entre outras: cirurgia geral, cirurgia pediátrica, urologia, ginecologia, obstetrícia, cirurgia vascular, neurocirurgia, otorrinolaringologia, cirurgia plástica, cirurgia torácica, cirurgia cardíaca, oftalmologia.

Assim, para otimizar o tempo no centro cirúrgico e diminuir a exposição de nosso paciente à intempérie do ambiente cirúrgico, torna-se necessária uma maior interação da equipe cirúrgica (cirurgiões, anestesiologistas, enfermeiros, técnicos de enfermagem, profissionais de transporte) no que diz respeito ao horário de chegada no centro cirúrgico e ao começo da anestesia e cirurgia. Some-se a isto a agilização da preparação do material cirúrgico-anestésico, que permitirá a realização de um procedimento com segurança numa instituição de ensino. Apesar de o tempo ter sido mais longo, não houve complicações de infecção no pós-operatório imediato nos pacientes incluídos na pesquisa.

É importante lembrar a preocupação do nosso hospital frente aos casos de infecção demonstrada no programa Hospital sem Sepses; preocupação não só do nosso, mas de tantos outros hospitais pelo país, personalizada na busca da acreditação por nossas instituições.

Essa acreditação norteia a busca pela qualidade e segurança tão almejada em nossas instituições. Prova disto é a preocupação da Organização Mundial deSaúde (OMS) com este assunto, que define a qualidade como: "o grau em que os serviços de saúde para indivíduos e população aumentam a probabilidade de resultados desejados e é consistente como o conhecimento profissional atual";; e a segurança como: "importante dimensão da qualidade que se refere ao direito das pessoas de terem o risco de um dano desnecessário associado ao cuidado de saúde reduzido a um mínimo aceitável". ${ }^{6}$ Assim, o binômio qualidade-segurança, quando colocado em prática, exige uma estratégia a ser utilizada na construção de sistemas de monitoramento com indicadores que medem várias dimensões da qualidade em conjunto (infraestrutura, exames complementares, satisfação do paciente e outros).

A anestesiologia não foge a essa regra. Prova disso são os indicadores desenvolvidos pela OMS para avaliar os padrões de qualidade no cuidado anestésico. Essa preocupação da anestesiologia com a segurança não é recente, oriunda de 1932 com a padronização das cores dos cilindros de gases medicinais. ${ }^{7} \mathrm{O}$ movimento em prol da segurança na sala de cirurgia tomou maior volume após um relatório americano lançado em 1999, intitulado "Errar é humano: construindo um sistema de saúde mais seguro". ${ }^{8}$

Esse cuidado se intensifica quando nos deparamos com uma lista de verificação (check-list), que funciona como anotações mentais e protocolos padronizados, induzindo o executor a uma série de ações prédeterminados que reduzam o mecanismo do erro. Partese da premissa que o tratamento anestésico-cirúrgico é complexo, envolvendo passos que devem ser relembrados e utilizados, por exemplo, na predisposição de sangramento pelo porte cirúrgico e reserva de sangue.

\section{Conclusão}

Com o desenvolver deste artigo, observamos que a nossa avaliação inicial não contemplava de maneira científica o conceito de qualidade e segurança em anestesiologia. Acreditávamos que, através deste questionário, teríamos um indicador fidedigno para propor mudanças, mesmo que insuficientes, mas de grande importância no atendimento anestésico-cirúrgico na nossa instituição.

Infelizmente, deparamo-nos com a insuficiência de indicadores abordados em nosso trabalho, os quais são muito mais complexos e abrangentes quando levantamos dados de segurança e qualidade na literatura. Aprimoramos nosso conhecimento e concluímos que para otimizar as cirurgias, reduzir o tempo e o mecanismo de erro e melhorar a qualidade ensino-aprendizagem no hospital é necessário o monitoramento constante através de indicadores, check-lists e vigilância.

Concluímos, portanto, que esses conhecimentos na prática são negligenciados na área da saúde como um todo, em especial na área anestésico-cirúrgica dos profissionais em especialização.

\section{Referências}

1. Biazzotto C, Brudniewski M, Auler J. Hipotermia no período per-operatório. Revista Brasileira Anestesiologia. 2006;56(1):89-06.

2. Nunes S, Alencar J, Gastão F. Anestésicos. Precondicionamento e Proteção Cerebral. Revista Brasileira Anestesiologia. 2013; 63(1):119-138

3. Amantea L, Teixeira M, Bertoncello K. Ocorrência de Hipotermia não planejada em sala de recuperação anestésica. Revista Brasileira de Anestesiologia, 2012.

4. Junior B, Scarpelini S, Rizoli S. Coagulopatia no trauma Medicina. Ribeirão Preto. 2007;40(4): 509-17.

5. Rocha JJR. Infecção em cirurgia e cirurgia das infecções.. Medicina. Ribeirão Preto; 2008. 41(4):487-90. 


\section{Artigo original}

6. Diego LAS, Cavalcanti IL, Cantinho FAF, et al. O Anestesiologista e o processo de acreditação hospitalar. Medicina perioperatória: pré-operatório, anestesia, reanimação e tratamento da dor. SAERJ. Rio de Janeiro; 2006.1001-1008.

7. Vecina Neto G, Malik AM. Gestão em Saúde. Rio de Janeiro: Guanabara Koogan; 2011.
8. Checklists save lives. Bull World Health Organ, 2008, 86:501-502

9. Jha Sumary of the Evidence of the patient safety: Implications for research. Geneva: WHO; 2008.

10. World Health Organization Safe surgery saves lives. [acesso em: 10 jul. 2012]. Disponível em: < http://www.who.int/patientsafety/safesurgery/en/ >. 